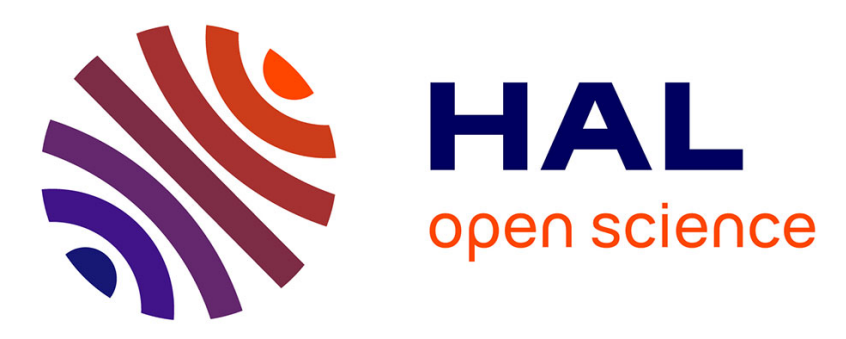

\title{
Reducing the impact of internal upsets inside the correlation process in GPS Receivers
}

\author{
Mohamed Mourad Hafidhi, Emmanuel Boutillon, Chris Winstead
}

\section{To cite this version:}

Mohamed Mourad Hafidhi, Emmanuel Boutillon, Chris Winstead. Reducing the impact of internal upsets inside the correlation process in GPS Receivers. Conference on Design and Architectures for Signal and Image Processing (DASIP 2015), Sep 2015, Cracow, Poland. 2015, Sep 2015, Krakow, Poland. hal-01211180

\author{
HAL Id: hal-01211180 \\ https://hal.science/hal-01211180
}

Submitted on 3 Oct 2015

HAL is a multi-disciplinary open access archive for the deposit and dissemination of scientific research documents, whether they are published or not. The documents may come from teaching and research institutions in France or abroad, or from public or private research centers.
L'archive ouverte pluridisciplinaire HAL, est destinée au dépôt et à la diffusion de documents scientifiques de niveau recherche, publiés ou non, émanant des établissements d'enseignement et de recherche français ou étrangers, des laboratoires publics ou privés. 


\section{Reducing the impact of internal upsets inside the correlation process in GPS Receivers}

\author{
Mohamed Mourad Hafidhi and Emmanuel Boutillon \\ Lab-STICC, UMR 6582, Université de Bretagne Sud \\ 56100 Lorient, France \\ email: mohamed.hafidhi@univ-ubs.fr, emmanuel.boutillon@univ-ubs.fr
}

\author{
Chris Winstead \\ ECE Dept., UMC 4120, Utah State University \\ Logan, UT 84322, USA \\ email: chris.winstead@usu.edu
}

\begin{abstract}
This paper ${ }^{1}$ examines two approaches to deal with internal logic upsets inside correlation process used in the tracking process of GPS receivers. These upsets can be produced due to process/voltage and temperature variations coupled with increased advancement of CMOS technology. If any upset occurs when computing the correlation function during each $10 \mathrm{~ms}$, then errors are propagated in tracking loops, resulting in a loss of the GPS signal tracking and a distorted position given by the receiver. Results of experiments using a GPS receiver design are presented in this paper to evaluate the performance of each method. The two proposed solutions (the Feedback freezing loop (FFL) and the Last Correct Value (LCV) methods) offer a big interest compared to the classical Triple Modular Redundancy (TMR) method since they provide the same performance as the TMR with low area complexity. This work can be extended to any system using feedback loops information.
\end{abstract}

\section{INTRODUCTION}

Efforts are actually made to minimize power consumption and maximize battery life in mobile devices. In contrast operating at minimal power levels can be a source of internal logic upsets resulting in momentary/persistent faults in the device's behavior. This problem increases with the advancement of CMOS technology [1] in combination with process/temperature and voltage (PVT) variations [2].

Global Positioning System (GPS) receivers are heavily used in mobile contexts, and there is motivation to minimize power consumption in these devices. Fault tolerance has been studied for data fusion in navigational systems that incorporate GPS receivers along with other sensor devices. For example, the authors of [3] consider an intelligent data fusion system to compensate for delayed or erroneous GPS data due to acquisition loss or signal obstruction. In [4] strategies have been proposed and evaluated for upset protection in Gold sequence generators in GPS receiver. [5] presents four approaches for upset protection in the Numerically controlled oscillator (NCO) carrier generator for GPS receiver. To track satellites' signals, GPS receivers has to determine over time the carrier frequency and the distance satellite-receiver [6]. This is accomplished by comparing a locally generated signals

\footnotetext{
${ }^{1}$ This work has received a French government support granted to the COMIN Labs excellence laboratory and managed by the National Research Agency in the "Investing for the Future" program under reference ANR-10LABX-07-01. It has also received support from the Brittany Region. Support was also from the US NSF award ECCS-0954747 and the Franco-American Fulbright Commission.
}

(Coarse/Acquisition codes and carrier) against the received signal, then moving smoothly in time local signals until it is time-aligned with the received signal. A correlation function is computed every $10 \mathrm{~ms}$ period to compare local signals with incoming signals. A maximum correlation output is achieved when the two signals are time aligned. Upsets when computing the correlation function can produce errors at its output. Because of feedback loops these errors will propagate over time and will corrupt the time estimation. Loss in the signal tracking process can be reported, forcing the receiver to restart the initial signal acquisition procedure. So it is increasingly important to deal with the impact of the upsets when they are generated in the correlation process.

The remainder of this paper is organized as follows. Sec. II gives detailed information in a compact form about GPS receiver architecture and rules of integrator modules. Sec. III describes some mechanisms to detect occurrences of upsets inside integrator modules. and Sec. IV presents the two proposed methods to reduce upsets' impact. Sec. V provides performance results, compares the probability for a receiver to be far from the reel (reference) position. Sec. VI offers conclusions.

\section{GPS SIGNAL PROCESSING}

A GPS is a well known technology that allows determining both the physical position and the absolute time of a receiver. The position in time and in space is determined thanks to a precise distance measurement with at least four GPS satellites. Each GPS satellite transmits a navigation message at 50-bits/s using the CDMA (Code Division Multiple Access) technology. The analytical expression of the transmitted signal of a satellite $a$ is:

$$
e_{a}(t)=c_{a}(t) d_{a}(t) e^{2 \pi j f_{L 1} t}
$$

where:

- $d_{a}(t)$ : navigation message of the $a^{t h}$ satellite,

- $c_{a}(t): a^{\text {th }}$ Coarse/Acquisition (C/A) satellite code with a Binary Phase Shift Keying (BPSK) modulation (i.e. $c_{a} \in$ $\{-1,1\})$,

- $f_{L 1}$ : the carrier frequency in the L1 GPS Band (Open Sevice).

GPS receiver has to demodulate the navigation message of different satellites in view to make the distance measurement. 


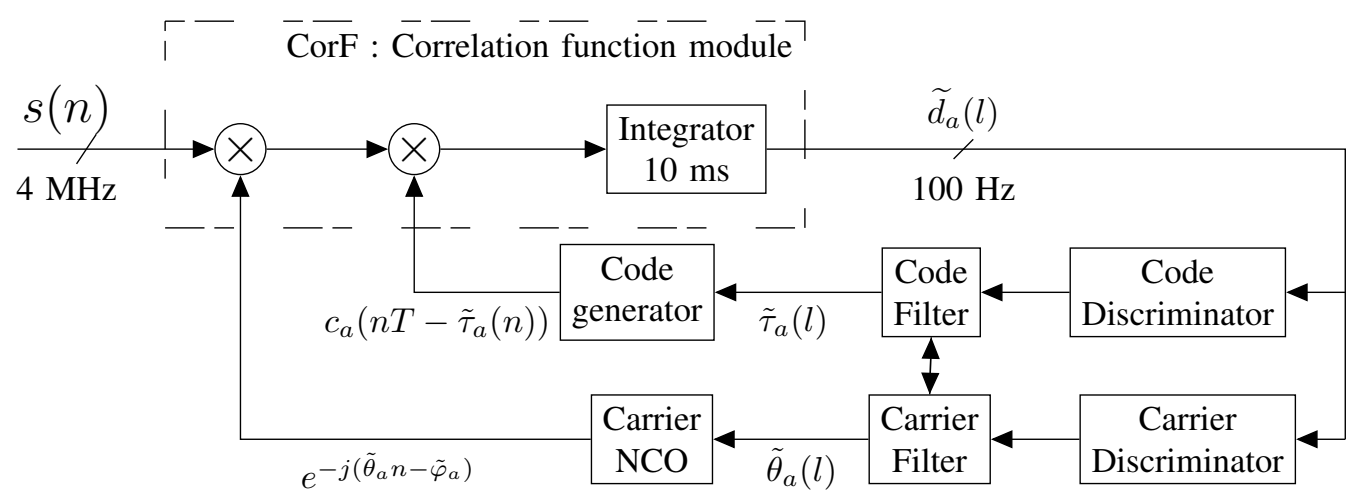

Fig. 1. Top Level of the simplified tracking channel module

This involves two essential and sequential process: the acquisition process and tracking process. The acquisition process is the process by which the receiver identifies which satellites are in view. It is a a three-dimensional search to determine the GPS satellite identifier (which is the index of its associated C/A code), the code phase (represented by $\tau$ ), and the carrier frequency offset due to Doppler effect (represented by $f_{d}$ ). Once estimated, the values of these parameters initiate the tracking modules. Since satellites are in continuous motion, the distance between any satellite and the receiver is dynamic. Besides to that, the carrier frequency of the received signal is also constantly changing in time due to Doppler shifts. Therefore, once acquired, GPS signals have to be tracked over time.

The GPS digital incoming signal is first demodulated by a frequency $F_{L 1}-F_{I}$ ( $F_{I}$ represent the intermediate frequency), and, after a pass band filter, sampled at a frequency of $4 \mathrm{MHz}$. The $n^{\text {th }}$ received sample $s(n)$ is given by:

$$
\begin{gathered}
s(n)=\sum_{k \in\{1 . . K\}} A_{k}(n T) d_{k}\left(n T-\tau_{k}(n T)\right) c_{k}\left(n T-\tau_{k}(n T)\right) \\
e^{j \theta_{k} n+\varphi_{k}}+w(n)
\end{gathered}
$$

where:

- $\theta_{k}=2 \pi T\left(f_{d(k)}(n T)+F_{I}\right)$,

- $A_{k}$ : Attenuation parameter of $k^{t h}$ satellite signal,

- $\tau_{k}$ : Propagation delay between satellite $k$ and receiver,

- $f_{d(k)}$ : Doppler frequency of satellite $k$,

- $\{1 . . K\}$ : Set of satellites visible at time $n$,

- $T$ : Sampling period $(T=0.25 \mu s)$,

- $w(n)$ : additive white gaussian noise.

As mentioned earlier the objectives of any tracking module is to extract the navigation message of the corresponding satellite. For each GPS satellite acquired, the GPS receiver associates a tracking channel module. A simplified representation of the channel tracking module is given in Fig. 1. Using estimated parameters $\tilde{f}_{d}$ and $\tilde{\tau}$ computed during the acquisition process, the GPS receiver produces, first, a local copy of the code $\mathrm{C} / \mathrm{A}$ and the carrier frequency. Then a correlation process between locally generated signals and incoming signals is launched to compute the correlation function between signals during each $10 \mathrm{~ms}$. C/A codes belongs to the family of Gold Pseudo Random Noise (PRN) sequences. Hence codes of all GPS satellites are orthogonal. Then, 10 ms-integrator output for the $a^{\text {th }}$ satellite is defined as,

$$
\tilde{d}_{a}(l) \triangleq \sum_{10 m s} s(n) c_{a}\left(n T-\tilde{\tau}_{a}(n T)\right) e^{-j\left(\tilde{\theta}_{a} n+\tilde{\varphi}_{a}\right)}
$$

Thus, a perfect time alignment $\left(\tilde{\tau}_{a}=\tau_{a}\right)$ and a perfect frequency and phase alignment $\left(\tilde{\theta}_{a}=\theta_{a}\right.$ and $\left.\tilde{\varphi}_{a}=\varphi_{a}\right)$ implies, according to (1) and (2), that $\tilde{d}_{a}(l)=d_{a}(l)$. The summation over $10 \mathrm{~ms}$ in (2) corresponds to the accumulation of 40,000 values (4 MHz clock during $10 \mathrm{~ms})^{2}$. Based on the integrator output (which represents the result of the correlation function during $10 \mathrm{~ms}$ ), discriminators and filters update the estimate Doppler frequency $\left(\tilde{f}_{d}\right)$ and the delay $(\tilde{\tau})$ in order to trade their evolution in time.

\section{UPSETS DETECTION METHODS}

Process/voltage/temperature (PVT) variations, coupled with continuous technology evolution and smaller transistor features have made internal logic upsets on devices more frequent. Logic upsets can manifest as momentary faults in the devices behavior, or as persistent faults. Occurrence of upsets can significantly affect the performance of circuits and can change output states. Therefore it is increasingly important to protect modern systems from upsets' impact to achieve acceptable reliability levels and maintain acceptable complexity and cost.

Detecting faults in combinatonational and sequential circuits is critical. Several techniques zre suitable to detect fault in combinatory circuit. First, the detection is done by the double modular redundancy (DMR) method. In this method the circuit is duplicated and a comparator is added to detect the incoherence between outputs of the two copies. In this case, area and power consumption increase $100 \%$. Besides to that DMR can detect upsets that affect only one copy.

To deal with these drawbacks, other design solutions for achieving transient-error detection were proposed. Detection

\footnotetext{
${ }^{2}$ Each bit of the navigation message have a duration of $20 \mathrm{~ms}(50 \mathrm{~Hz}$ bit rate). Thus, assuming perfect bit timing synchronisation, $d_{a}(l)$ is constant while computing (2)
} 


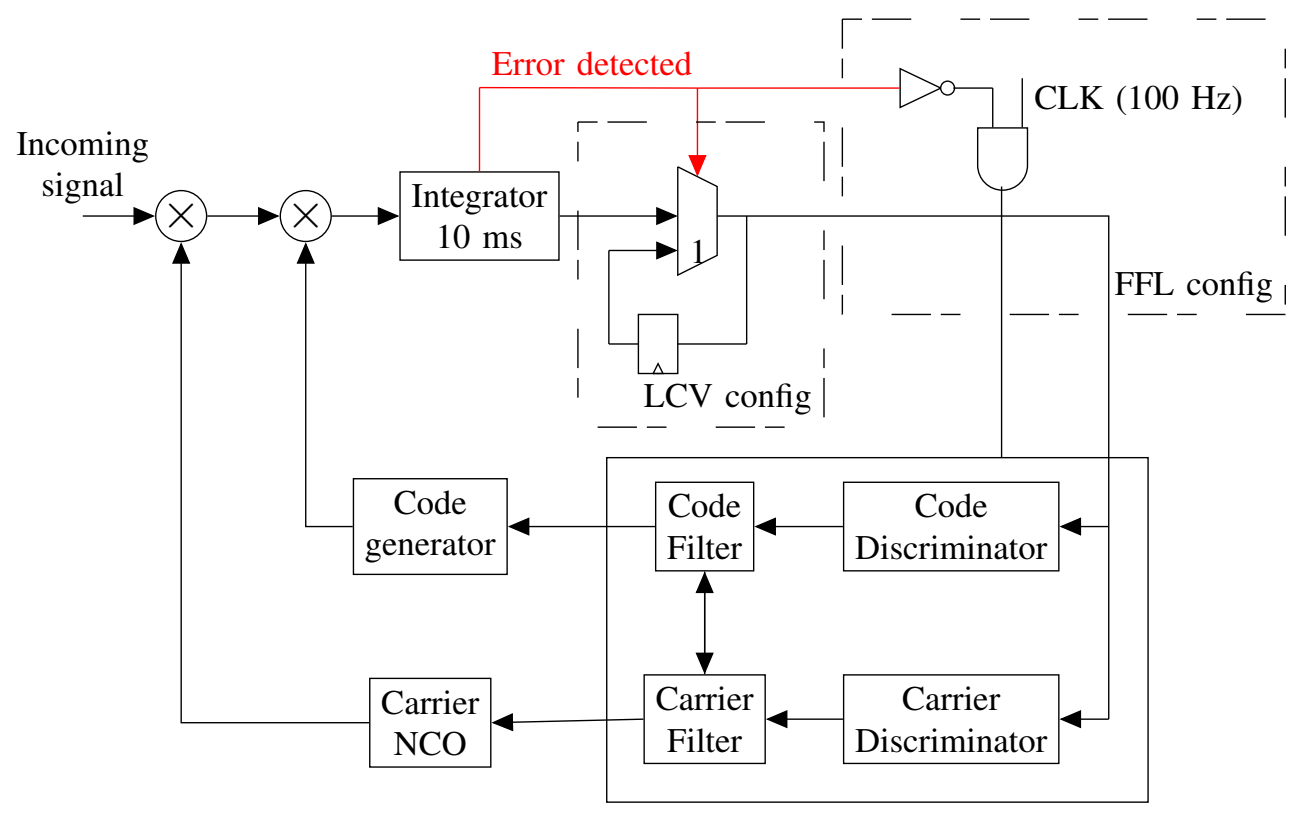

Fig. 2. Configuration of proposed method. We add to the original tracking channel either the FFL or the LCV configuration

can be realized by the double sampling method cited in [7] and [8]. The main idea of this method is to take advantage of the temporal nature of transient faults, and achieve transient faults detection by using time redundancy. As shown in fig. 3, transient faults detection is realised by observing the output signals of a given circuit at two instants (double sampling). The schema is composed of:

- A latch or flip-flop at the output of the circuit to make a delay version of the input signal.

- A comparator to check the state of the original and the delayed versions.

- An error detection flip-flop using as latching event the rising edge of the clock.

Simulations and performances are presented in [9]. Results show that this new method uses a low hardware cost to guarantee a complete timing error and very soft errors detection. The upsets' detection can also be achieved by bulk built-in current sensors (BICS) [10], [11]. Based on the same approach (comparing the output at two different instant), BICS detect the ionisation current in the bulk by sensing the current. Two BICS are used in any solution to detect upsets: PBICS and NBICS. PBICS is used to detect output transitions from 0 to 1 and PBICS to detect output transitions from 1 to 0 . Solutions based on the BICS approach has been proposed to make the detection in sequential and in combinational logic. The BICS appears in [12] and [13] where a memory cell and 4-bit multiplier are respectively circuit under test. As for the double sampling method, with BICS approach we can also reduce area and power dissipation penalties compared to the DMR. To summarize, many are upsets' detection method that can be used to detect occurrence of upset in any application. In our case we will focus only on how we can limit upsets' impact after detecting their presence.

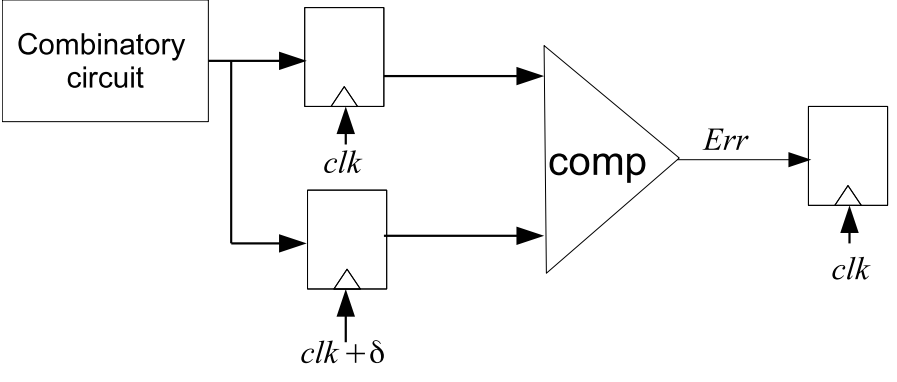

Fig. 3. Double sampling approach [8]

\section{LIMIT UPSETS' IMPACT INSIDE CORRELATION PROCESS}

In this section, we first define the method to assess the quality of a GPS with a noisy integrator. Then, assuming that error can be detected (see previous section), we define two methods to reduce efficiently the impact of errors.

\section{A. Quality of a noisy GPS receiver}

The methodology used to evaluate the quality of the proposed method is three fold:

- Use the front end of a GPS receiver to store incoming GPS signal $s(n)$ over a significant period of time (few minutes).

- Process the stored input GPS signal with a reference noiseless GPS receiver [14] to generate the set of successive estimated positions $X(p)_{p=1 \ldots Q}$ (The reference position).

- Replace the noiseless GPS receiver with a noisy GPS receiver and process a Monte-Carlo simulation processing $N$ times the stored incoming GPS signal to generate sets of noisy position $\tilde{X}^{i}(p)_{p=1 . . Q}, i=1 . . N$. 
Then, we can study the link between the model of error injection, the hardware used to mitigate errors and the statistical difference between GPS noiseless position $X$ and the GPS noisy position $\tilde{X}$.

1) Model of noise: The model of noise used in this paper is very simple. For each active tracking module, every output of the integrator is assume to be exact with a probability $(1-p)$ or to be faulty with a probability $p$. In case of faulty result, a random value is uniformly drawn between the smallest and highest value of $\tilde{d}_{k}(l)$ to replace the exact value.

2) Metric to evaluate the quality of the noisy GPS receiver: Two metrics are used to evaluate the quality of the noisy GPS receiver. The first one is the mean squared error (EQM) defined by:

$$
E Q M(p)=\sqrt{E\left(|X-\tilde{X}|^{2}\right)}
$$

where $p$ is the probability of the upsets' injection model (noise) at the output of the integrator $10 \mathrm{~ms}$. This parameter gives us an idea about the distance, on average, we will be far from the reference position. The second parameter is:

$$
f(d, p)=\operatorname{Pr}(|X-\tilde{X}|>d)
$$

Given the probability of error injection, $p, f(d, p)$ reflect the probability that the noisy GPS receiver compute a position at a distance $d$ from the reference position.

\section{B. Feedback freezing loop (FFL) method}

The first method we propose is to freeze the feedback loops once we detect occurrence of upset. The idea is that it is less harmful to inject nothing in the feedback loop than a wrong value. Fig. 2 illustrates the configuration used in this method. So if we detect that upsets has been produced during the 10 ms period $\left(T_{1}\right)$, feedback loops are frozen (or blocked) until a new computation of the correlation function is achieved (i.e until the 10 next millisecond $T_{2}$ ). Code and carrier generators will use the same codes and carrier that have been generated during the previous $10 \mathrm{~ms}$ period $\left(T_{0}\right)$. With this approach we can guarantee that errors in the correlation process don't propagate over time. Fig. 2 describes the configuration used for this method.

\section{Last Correct Value (LCV) method}

The second proposed method uses the last correct value of the integrator output to feed the feedback loop once we detect that an error has been produced when calculating the correlation at the next $10 \mathrm{~ms}$-period $T_{1}$. Fig. 2 describes the configuration used for this method. If $\tilde{d}_{a}(l)$ is the result of the correlation function at time step 1 , at time step $l+1$ and if errors have been produced when computing $\tilde{d}_{a}(l+1)$, the system uses the value $\tilde{d}_{a}(l)$ to be sent to feedback loops instead of $\tilde{d}_{a}(l+1)$.

\section{Triple modular redundancy (TMR)}

TMR is a classical solution for fault tolerance in electronic systems [15]. It has been widely used for electronics in the space and nuclear industries to ensure reliable operation [16]. In a TMR system, the original module (the correlation function computaion (CorF) module) is replicated three times, and error correction is achieved by a majority vote operation. We note that there are several configurations, such as one-voter TMR, the triple-voter TMR or the restorative-feedback TMR [17]but to minimize overhead we only consider the most basic TMR strategy as represented in fig. 4. If an upset occurs in any one of the three CorF modules, the other two CorF can correct and mask the fault assuming that the vote operation is not faulty. However once the voter is faulty, errors can be generated. These errors will propagate over time resulting in a fault at the output of the integrator $10 \mathrm{~ms}$ in Fig. 1.

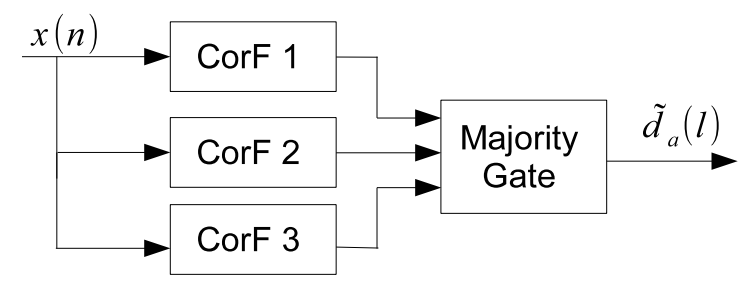

Fig. 4. TMR strategy of protection

In the next section, simulation results show the quality of the two proposed methods compared to the classical TMR.

\section{Performance Results}

All of the described methods were evaluated in term of EQM and Probability of an alignment of the reference position as defined in (3) and (4). Although they are simpl to implement and with a very low cost area, LCV and FFL methods offers a high gain performance. Fig. 5 compares EQM of different methods. In this figure, $E Q M=4.6510^{6} \mathrm{~m}$ (which represent the reference position) is equivalent to a loss of the GPS signal tracking. As we can see in Fig. 5, for a probability of upsets up to $p=0.4$, LCV method gives an error in the position that does not exceed on average $2 \mathrm{~m}$. Thus the LCV methods permit to achieve an acceptable reliability even with high upset probability. Fig. 6 and Fig. 7 show the variation of this probability as a function of the distance between the noisy and the reference position when $p=0.1$ and $p=0.2$ respectively. For instance when $p=0.1$, the probability of been $1 \mathrm{~m}$ far from the reference position goes from 0.6 to 0.009 using the LCV method.

\section{CONCLUSION}

Our results show an improvement in the probability of the removal from the reference position and in term of EQM with the LCV and FFL methods. Up to a probability of upset $p=0.4$, with LCV configuration we guarantee an acceptable reliability (EQM ; $2 \mathrm{~m}$ ) for a low hardware complexity compared to the TMR strategy. 


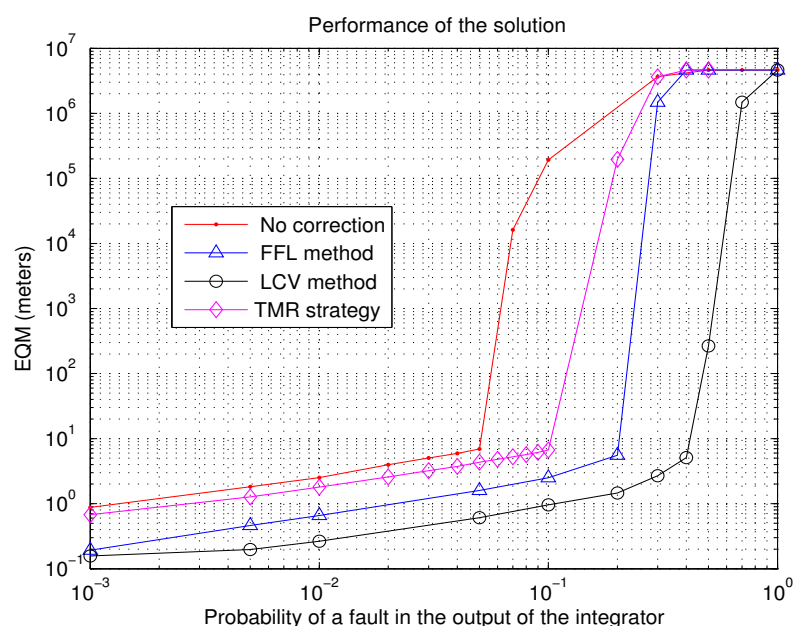

Fig. 5. $E Q M=f(p)$; When $p=10^{-1}$, EQM goes from $1000 \mathrm{~km}$ to an orror in the position of about 1 meters (using FFL method) and few centimeters (with LCV method).

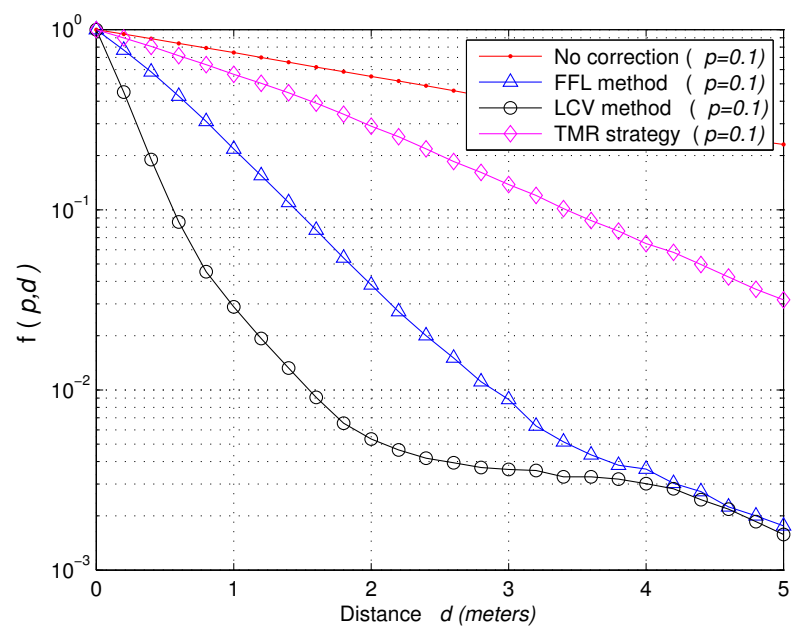

Fig. 6. The probability of the removal (in meters) from the reference position when the upset probability $p=0.1$

\section{REFERENCES}

[1] P. B. J. A. Jayanth Srinivasan, Sarita V. Adve, "The impact of technology scaling on lifetime reliability," The International Conference on Dependable Systems and Networks (DSN-04), 2004.

[2] S. Borkar, "Tackling variability and reliability challenges," IEEE Design and Test of Computers, vol. 23, no. 6, 2006.

[3] M. Jaradat and M. Abdel-Hafez, "Enhanced, delay dependent, intelligent fusion for ins/gps navigation system," Sensors Journal, IEEE, vol. 14, no. 5, pp. 1545-1554, May 2014

[4] C. W. Mohamed Mourad Hafidhi, Emmanuel Boutillon, "Reliable gold code generators for gps receivers," The International Midwest Symposium on Circuits and Systems (MWSCAS 2015), 2015.

[5] C. W. Mourad Dridi, Mohamed Mourad Hafidhi and E. Boutillon, "Reliable nco carrier generators for gps receivers," submitted in Design and Architectures for Signal and Image Processing (DASIP 2015), 2015.

[6] J. B.-Y. Tsui, Fundamentals of Global Positioning System Receivers. Wiley-Interscience, 2000.

[7] M. Nicolaidis, "Time redundancy based soft-error tolerance to rescue nanometer technologies," 17th IEEE Proceedings. VLSI Test Symposium, 1999, 1999.

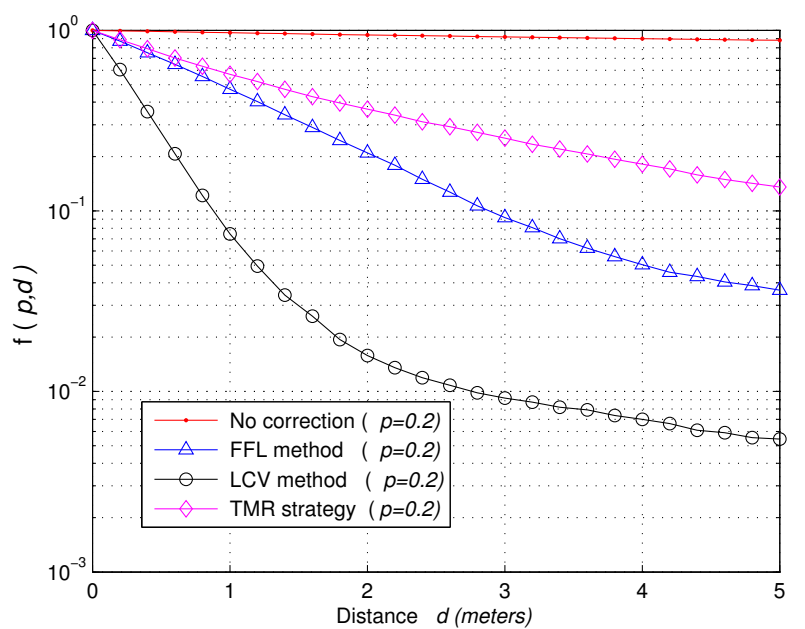

Fig. 7. The probability of the removal (in meters) from the reference position when the upset probability $p=0.2$

[8] - "Circuit logique protege contre des perturbations transitoires," Patent WO2000054410 A1, Mar. 9, 2000, 1999.

[9] M. N. Lorena ANGHEL, "Cost reduction and evaluation of a temporary faults detecting technique," DATE 'OO Proceedings of the conference on Design, automation and test in Europe, pp. 591-598, 2000.

[10] M. N. F. Vargas, "Seu-tolerant sram design based on current monitoring," Fault-Tolerant Computing, 1994. FTCS-24. Digest of Papers., TwentyFourth International Symposium on, pp. 106 - 115.

[11] F. W. C. P. S. G. Balkaran Gill, Michael Nicolaidis, "An efficient bics design for seus detection and correction in semiconductor memories," European design and Automation Association, DATE05, Mar 2005.

[12] F. S. Daniel Gomez Toro, Matthieu Arzel and M. Jzquel, "Soft error detection and correction technique for radiation hardening based on c-element and bics," Circuits and Systems II: Express Briefs, IEEE Transactions on, vol. 61, no. 12, pp. 952 - 956 .

[13] M. V. G. W. F. L. K. Egas Henes Neto, Ivandro Ribeiro, "Using bulk built-in current sensors to detectsofterrors," Micro, IEEE, vol. 26, no. 5, pp. $10-18$.

[14] A. Dion, Personal communication. Institut Supérieur de 1' Aéronotique et de 1' Espace (ISAE)., 2014.

[15] R. E. Lyons and W. Vanderkulk, "The use of triple-modular redundancy to improve computer reliability," IBM J. Res. Dev., vol. 6, pp. 200-209, 1962.

[16] Y. Z. Yimao Cai and L. La, "Implementation of a reconfigurable computing system for space applications," System Science, Engineering Design and Manufacturing Informatization (ICSEM), vol. 2, 2011.

[17] C. Winstead, A. Tejeda, E. Monzon, and Y. Luo, "Error correction via restorative feedback in M-ary logic circuits," J. of Mult. Valued Logic and Soft Comp., vol. 23, no. 3-4, 2014. 